\title{
Discussion on the Application of Zero Translation Theory in Translation of Network Popular Words
}

\author{
Liang $\mathrm{Wei}^{1, \mathrm{a}}$ \\ ${ }^{1}$ Jiangxi Institute of Economic Administrators, Nanchang, Jiangxi, 330088
}

Keywords: Network Popular Words; Zero Translation Theory; Application

\begin{abstract}
With the rapid development of network information technology, the network platform provide a virtual space for people to communicate and communicate, and the network buzzwords will rise. The continuous development of economic globalization has promoted the development of cross-cultural communication, thus put forward new requirements for the translation of network popular words. This paper discusses the characteristics of network popular words, the definition and classification of zero translation theory and the application of zero translation theory in network popular words.
\end{abstract}

\section{Introduction}

Network popular words are Internet users in order to facilitate the chat, to create a humorous chat atmosphere and convention, is the continuous development of network information technology reflects. Internet pop words with simple, novelty, euphemism, contingency and other characteristics, it is the network pop words of these characteristics to the translation of the network word has brought some difficulty [1]. In order to solve the problems existing in the translation of network buzzwords, the translator can use the zero translation concept to translate, the absolute zero translation method and the relative zero translation of the specific methods have a high value, according to the different nature of the word to take different zero Translation method.

\section{The Characteristics of Network Popular Words}

Network popular words are generated with the rapid development of the network, and through the form of network platform to spread to people's real life. Internet popular words and traditional language vocabulary compared to the following highlights: simple, such as "88" said goodbye, with "520" that "I love you", "sb" that "sucker"; novel "With you" means "like you", with "sauce purple" that "this way", with "silver home" that "people; euphemism, such as" dinosaur "on behalf of ugly girls, with" Frog "on behalf of the ugly boys; accidental, many popular words are netizens whim, with chance; lack of rules, many network pop words appear to facilitate chat, create a humorous chat atmosphere, rarely meet Standard vocabulary requirements.Although the network of popular words have penetrated into people's lives, but because of its own characteristics of the network pop words have irreducible features, the use of zero translation theory to reduce the use and expression between the various languages The difference in the way is wise.

\section{Definition and Classification of Translation Theory}

Zero translation is not without translation, but rather does not use the words in the target language to translate the words in the original language, with the following two meanings: the first layer meaning is intentionally not translated words in the original sentence; Refers to the words in the original sentence without the existing words in the target language. For example, in the article published in the Times, the term "urban management", which is often discussed by the Chinese people, is mentioned in the article, which is the word "chengguan- urban security guards" which translates the term "urban management". This kind of refueling translation method is zero translation method, because urban management is as a network of popular words appear in the report which can not be directly translated [2]. Zero translation can be divided into two categories: 
absolute zero translation and relative zero translation. Absolute zero translation can be divided into the provincial translation and translation method, relative zero translation can be divided into transliteration, induction and compensation law. Even if there is no standard answer to the definition of zero translation theory, it has been applied to the translation of network buzzwords and has been recognized by people. It is worth noting that translators should choose which zero translation method to use when translating network buzzwords based on the needs of the target population and the meaning of words in the original statement.

\section{The Application of Triple Translation Theory in Network Popular Words}

Above mentioned to mention the absolute zero translation can be divided into the provincial translation and translation method. Provincial translation is a special alternative translation method, in today's world of cross-cultural communication, for the differences between cultures and languages, the use of literal translation is not feasible. In order to solve this problem, people use the provincial translation instead of literal translation. As the name suggests, it is appropriate to omit some of the words in the original sentence, without changing the meaning of the original sentence. For example, everyone is familiar with the curse statement: You are a fool, is a 290 (250,38,2 three combinations). In China, often use 250,38 and 2 to describe a person stupid, and in the English translation did not reflect, only "comepletedongkey" to express the meaning of the original sentence.

The "we" on the earth, the water is larger than the land area, where the "we" pronoun is not translated. The horse is a useful animal. Translated into "horse is a useful animal", omitting the pronoun "The" in the original sentence.

Translation method refers to the original sentence in the network of popular words directly to the target language, such as we often say that the T-shirt is from the English word "T-shirt" translated from the BF is also through the word "boy friend" directly Translated over. The gross domestic product (GDP), which is of concern to the whole country, is translated from the English "Gross Domestic Product". There are some of the most common well-known radio and television stations referred to, CCTV, BBC, CNN, BTV and so on.

Relative zero translation refers to the translation of some of the words in the original sentence. It includes transliteration, phonetic translation, translation of translation, translation, translation and literal translation.

Transliteration refers to a word in the original sentence pronunciation to translate into the target word, is an effective way to solve the words can not be translated. Such as the English word "kungfu" is through the transliteration of the "Kung Fu" translation, "tofu" is also the use of transliteration translation. Although the transliteration on the surface looks very simple, is a no technical content of the translation method, but the translation of the vocabulary involved in the characters and events should be people understand the event or people, or people difficult to understand, What effect. For example, "Satsuki" was translated into "xiaoyueyuue", "Zengge" was translated into "Zengge", "Chunge" was translated into "chunge" [3].

Phonetic translation method refers to a part of the pronunciation of words according to the translation, a part of the translation according to the meaning of words, so that people can better understand and accept. For example, to force the word is translated into "gelivable", which "geli" is to force the transliteration, "yes" is the English word adjective suffix. Such as foreigners will be on the network very famous "Xifeng" translated into "Lady Fengfeng" through the combination of sound and sound law, so that Xifeng exclusive words can easily let people understand. In recent years, the term "base friend" is often used to describe the feelings of friends is very good, in fact, "base friends" is the word through the combination of sound and meaning translation, the "Gay" pronunciation of the first letter "G "As the" base friends "transliteration part of the" Gay "connotation meaning as part of the translation.

Compensation translation method refers to the pronunciation of the target language in the original sentence to find the same pronunciation words, but the word should have a certain meaning in order to compensate for the translation process caused by the loss. Such as many people love to 
drink in the summer Coca-Cola, is through the English word "Coca Cola" compensation translation over. Coca-Cola's translation is not only similar to the pronunciation of English words, but also to attract customers to buy the purpose. Such as people are longing for the purchase of Mercedes-Benz, is derived from the word "Benz" in the "Ben" pronunciation, but the Mercedes-Benz word itself has an elegant, fast meaning, can make up for the loss caused by the translation process.

Translation is the use of the target language in the shape of the word to express the original sentence with a shape of the image of the method. The importance of translation is that the original sentence and the target language have a corresponding shape image. As we often say "triangular relationship" can be translated into "T-relashionship". "The road is tortuous" can be translated into "The road ahead is zigzag".

Domestication translation method refers to the words in the target language to find the same meaning in the original words, but the use and expression of the habit to meet the target language. For example, the network of popular words "is represented", in English, there is no such usage and expression of habits, we need to use the translation of naturalization translation method, translated into "voters without voting rights." Only use the translation of natural translation On behalf of "foreigners can better understand the" representative "of the meaning of the meaning. "High-speed rail" translated as "just-in-the-official-date almost brought by the Hight Speed Train".

Literal translation method refers to the direct translation of the annotation in order to allow readers to better understand the meaning of words, such as "Hamburger" translated into hamburgers, hamburgers which "package" is to raise, help people understand the hamburgers The

People like to use "God horse is clouds" to express their attitude to life, which "clouds" can be translated as "floating clouds", then add "refers to something that is nothing but nothing", you can be very good Let foreigners understand the true meaning of "clouds". There are a few years ago popular QQ game "steal food", "stealing food" can be translated as "stealing vegetables", plus a "popular game that people can get joys from stealing their e-pals' vegetables on The key to the use of the literal translation method is to raise the link, and the use of the supplement can make the reader more intuitively understand the network buzzword.

\section{Conclusion}

In short, the use of zero translation theory plays an important role in the translation of network buzzwords. Not only for the translator to provide different translation methods and ideas, but also to solve the current problems of online vocabulary translation and network vocabulary is not translatable. With the continuous development of economic globalization and the continuous development of network information technology, network popular words will be more and more people with the demand, zero translation theory will be used more and more widely.

\section{References}

[1] Zhang Yan, Guo Xiao. Zero translation theory in the use of network popular words translation [J]. Modern communication, 2012, (01): 43-44.

[2] Wang Jing. Zero translation strategy in network popular words [J]. Development, 2016, (32): 232.

[3] $\mathrm{Hu}$ Anna. The basis of the translation of hot words under zero translation [J]. Journal of Xiangyang Vocational and Technical College, 2016, (05): 65-68. 